(c) American Dairy Science Association, 2006.

\title{
Evaluation of Models to Describe Ruminal Degradation Kinetics from In Situ Ruminal Incubation of Whole Soybeans
}

\author{
M. H. Fathi Nasri, ${ }^{*}$ M. Danesh Mesgaran, ${ }^{*}$ J. France, $†$ J. P. Cant, $†$ and E. Kebreab ${ }^{1}$ \\ *Department of Animal Science, Faculty of Agriculture, Ferdowsi University of Mashhad, Mashhad, Iran 91775-1163 \\ †Centre for Nutrition Modelling, Department of Animal and Poultry Science, University of Guelph, Guelph, Ontario N1G 2W1, Canada
}

\begin{abstract}
Different mathematical models were evaluated as candidates to describe ruminal dry matter (DM) and crude protein $(\mathrm{CP})$ degradation kinetics of raw and roasted whole soybeans from data obtained using the in situ polyester bag technique. Three models were used: segmented with up to 3 straight lines (model I), negative exponential (model II), and rational function or inverse polynomial (linear over linear; model III). A fourth, a generalized sigmoidal model, was also considered but the data did not exhibit sigmoidicity, so it was dropped from the analysis. Lagged and nonlagged versions of each model were fitted to the DM and CP disappearance curves of 6 different feeds ( 2 cultivars of raw or differently heat-processed whole soybean). The comparison between lagged and nonlagged versions of each model, based on statistical and behavior characteristics, showed for all models that the discrete lag parameter did not significantly improve the fit to ruminal DM and CP disappearance curves. The comparison between models (using nonlagged equations) showed that models I and II gave better goodness-of-fit than model III. Based on biological characteristics, models II and III underestimated the undegradable DM and CP fractions, but there was no significant difference between models for extent of degradation.
\end{abstract}

Key words: mathematical model, ruminal degradability, in situ incubation, degradation kinetics

\section{INTRODUCTION}

In vivo and in situ incubation of feeds in the rumen serves as a basic procedure in many feed evaluation systems and the formulation of hypotheses concerning underlying biological concepts has led to development of different mathematical models describing the resultant time course disappearance curves of feed fractions. The use of models allows comparison of parameter esti-

Received December 9, 2005.

Accepted March 15, 2006.

${ }^{1}$ Corresponding author: ekebreab@uoguelph.ca mates (or combinations thereof) that ideally reflect these biological concepts, between feeds or feeding systems. Degradation and passage parameters are important aspects of rumen models (e.g., NRC, 2001; Kebreab et al., 2004; Thomas, 2004); therefore, accurate estimates of degradation parameters are required for incorporation into these systems. A number of methodological factors affecting the experimental measurements of in situ disappearance of feed samples has received due attention (Nocek, 1988; Huntington and Givens, 1995), but much less attention has been paid to the choice of mathematical model to fit the curves and goodness-of-fit of the model. In the present study, 3 different mathematical models (Ørskov and McDonald, 1979; France et al., 1990; and Lopez et al., 1999), which were also used to evaluate gas production profiles in a reparameterized form (France et al., 2005), were selected. A fourth model (a generalized sigmoidal function) was also tested. The objective of the study was to evaluate use of these models to determine DM and CP degradability parameters of raw and roasted whole soybean samples for fitting ability.

\section{MATERIALS AND METHODS}

\section{Samples and Analyses}

Two Iranian cultivars of soybeans (Sahar and Williams), raw, roasted, and steep-roasted, and considered for evaluation of heat-processing effects on DM and CP ruminal degradability in another experiment, were used in this study. For heat processing of soybean seeds, they were fed into a turning cylindrical tunnel $(50 \mathrm{~cm}$ in diameter, turning at a speed of 2.5 cycles per minute) with a flame and blower at its end, so that the seeds were exposed to burning air. The seeds traversed the cylinder tunnel (4 m long), so that the temperature of the beans exiting the roaster was 130 to $135^{\circ} \mathrm{C}$. Then, some seeds were gradually cooled (about $1 \mathrm{~h}$ ), and the rest were immediately placed in isolated barrels without cooling, and covered with canvas for about $45 \mathrm{~min}$ (steeping), and then cooled.

Dry matter and CP of samples ( 6 feeds) before and after incubations were determined using a forced-air 
Table 1. Dry matter and CP content (g/kg of DM; SD in parentheses) of heat-processed Sahar and Williams cultivars of soybean seeds

\begin{tabular}{|c|c|c|c|c|c|c|}
\hline & \multicolumn{3}{|c|}{ Sahar } & \multicolumn{3}{|c|}{ Williams } \\
\hline & Raw & Roasted & Steep-roasted & Raw & Roasted & Steep-roastec \\
\hline DM & $920(9.5)$ & $970(9.0)$ & $975(9.9)$ & $910(10.1)$ & $985(9.0)$ & $970(12.2)$ \\
\hline $\mathrm{CP}$ & 369 (21.9) & $381(25.0)$ & 378 (23.1) & $356(28.1)$ & $376(37.5)$ & $375(22.0)$ \\
\hline
\end{tabular}

oven at $96^{\circ} \mathrm{C}$ for $48 \mathrm{~h}$, and the Kjeldahl method (Kjeltec 2300 Autoanalyzer, Foss Tecator AB, Hoganas, Sweden), respectively (Table 1).

\section{In Situ Procedure}

Two ruminally fistulated $(430 \pm 10 \mathrm{~kg})$ Holstein steers fed a TMR twice daily ( 0900 and $1600 \mathrm{~h}$ ) were used for incubation of samples. The TMR included (on a DM basis) $2.7 \mathrm{~kg}$ of alfalfa hay, $1.75 \mathrm{~kg}$ of corn silage and $2.25 \mathrm{~kg}$ of concentrate (barley 63.5, cottonseed meal 5.8, beet pulp 17.3, wheat bran 10.0, limestone 1.0, salt 0.4 , vitamin-mineral supplement 0.5 , and urea $1.5 \%$ ) per steer per day. To determine the DM and CP degradability coefficients, $5 \mathrm{~g}$ of DM equivalent of each sample (ground using 2-mm screen mill) was placed in individual polyester bags (made of artificial silk cloth with a $50-\mu \mathrm{m}$ pore size and averaging $12 \times 19 \mathrm{~cm}$ ). Bags were placed in the dorsal sac of the reticulorumen of each steer after the $0900 \mathrm{~h}$ feeding. Replicate bags of each feed sample were removed at 1, 2, 3, 4, 8, 16, 24, 36, 48 , and $72 \mathrm{~h}$ of incubation and were hand washed thoroughly in cold running water until the rinsing water was clear. Two bags of each sample were washed without incubation in the rumen (0-h samples). The bags were dried in a forced-air oven $\left(58^{\circ} \mathrm{C}, 48 \mathrm{~h}\right)$ and weighed to determine DM disappearance. The residues were analyzed for $\mathrm{N}$ content. Degradability of DM and CP was recorded at each incubation period for each of 6 feeds, yielding a total of 12 disappearance curves for each model.

\section{Mathematical Models}

Table 2 shows the 3 models, 1 piecewise linear and 2 diminishing returns, used to describe ruminal degradation of DM and CP of the samples. Model I is a segmented model with 3 spline-lines delimited by 2 nodes or break points, constraining splines 1 and 3 to be horizontal asymptotes, and follows zero-order degradation kinetics (France et al., 1990). Model II is a negative exponential equation (monomolecular or Mitscherlich) assuming first-order kinetics and a constant fractional rate of degradation (Ørskov and McDonald, 1979). Model III is a rational function or inverse polynomial (linear over linear), which describes a rectangular hyperbola and assumes first-order kinetics with a variable fractional rate of degradation that declines with time (Lopez et al., 1999). This latter model is akin to the Michaelis-Menten equation of enzyme kinetics. A discrete lag parameter $(L)$ was included in each model to represent the time interval before degradation commences. A reparameterized and generalized sigmoidal model, the Richards (Thornley and France, 2006), was also used to test whether the degradation profile could be better described using an S-function. The Richards equation was chosen because it encompasses the Gompertz, logistic, and monomolecular (diminishing returns) when its additional parameter $(n)$ has a value of 1,0 and -1 , respectively. In all of the profiles fitted, $n$ was not significantly different from -1 ; therefore, sigmoidal analysis was dropped and piecewise linear and diminishing return models were used with and without a lag parameter.

Each model was fitted to the DM and CP disappearance curves by nonlinear regression using the PROC NLIN of SAS (SAS Institute, 1999) to estimate ruminal degradation parameters. Several possible starting values were specified for each parameter, so that the NLIN procedure evaluated the model at each combination of initial values on the grid, using for the first iteration of the fitting process, the combination yielding the smallest residual sum of squares. Rapidly soluble fraction $(a)$, slowly degradable fraction $(b)$, degradation rate constant (c), or time constant $(T)$, and $L$ were estimated for each disappearance curve using each model. The extent of degradation $(E)$, for a given passage rate $(0.06$ and $0.08 / \mathrm{h}$ ), was calculated from these estimated parameters as shown in Table 2 . In calculating $E$, the washout fraction was assumed to be completely degradable; however, $E$ can be readily calculated based on other assumptions about degradability of the washout fraction as described by Dhanoa et al. (1999). In model III, for calculating $E$, the function

$$
a+b k \int_{L}^{\infty}\left(\frac{t-L}{t-L+T}\right) \mathrm{e}^{-k t} \mathrm{~d} t
$$

was coded in the advanced continuous simulation language ACSL (Aegis Simulation, Inc., Huntsville, AL) 
Table 2. Candidate models for describing disappearance curves ${ }^{1}$

\begin{tabular}{lllll}
\hline & $\begin{array}{l}\text { Fractional } \\
\text { degradation } \\
\text { rate, } \mu(\mathrm{h})\end{array}$ & $\begin{array}{l}\text { Disappearance } \\
\text { to time } t, p(\%)\end{array}$ & Nodes & $\begin{array}{l}\text { Extent of degradation, } \\
\text { Model }^{2}\end{array}$ \\
\hline I & $\frac{c}{b-c(t-L)}$ & $a+c(t-L)$ & $L \leq t<L+\frac{b}{c}$ & $a+\frac{c}{k} \ln \frac{k b+c \mathrm{e}^{k L}}{c}-c L$ \\
II & $c$ & $a+b\left[1-\mathrm{e}^{-c(t-L)}\right]$ & $L \leq t$ & $a+\frac{b c \mathrm{e}^{-k L}}{c+k}$ \\
III & $\frac{1}{t-L+T}$ & $a+b \frac{t-L}{t-L+T}$ & $L \leq t$ & $a+b k \int_{L}^{\infty}\left(\frac{t-L}{t-L+T}\right) \mathrm{e}^{-k t} \mathrm{~d} t$ \\
\hline
\end{tabular}

${ }^{1} a=$ rapidly soluble fraction $(\%) ; b=$ slowly degradable fraction $(\%) ; c=$ degradation rate constant $(\% / \mathrm{h}$, model I; /h, model II); $k=$ fractional passage rate $(/ \mathrm{h}) ; L=$ lag time $(\mathrm{h}) ; T=$ time constant $(\mathrm{h})$, such that $L$ $+T$ is the time taken for half of the slowly degradable fraction to disappear; $t=$ time since start of incubation (h).

${ }^{2}$ Model I, zero-order degradation (France et al., 1990); model II, first-order degradation, constant $\mu$ (Ørskov and McDonald, 1979); model III, first-order degradation, variable $\mu$ (Lopez et al., 1999).

and solved using a fourth-order Runge-Kutta algorithm with an integration interval of $0.1 \mathrm{~h}$.

\section{Statistical Analyses}

Statistical comparison between lagged and nonlagged versions of each model to test whether the additional parameter $(L)$ improved the fit was performed by $F$-test using the following equation (Motulsky and Ransnas, 1987):

$$
F=\left[\left(\mathrm{SS}_{1}-\mathrm{SS}_{2}\right) /\left(\mathrm{df}_{1}-\mathrm{df}_{2}\right)\right] /\left(\mathrm{SS}_{2} / \mathrm{df}_{2}\right)
$$

where SS is the sum of squares, and $\mathrm{df}$ is the number of degrees of freedom. The subscript 1 refers to the fit with fewer parameters, the nonlagged version. Furthermore, the $L$ parameter was tested to see if it was significantly different from zero. Therefore, after fitting the lagged version of each model to the disappearance curves, the average of parameter $L$ was calculated for each model and its difference from zero tested ( $t$-test). To evaluate the ability of each model (both versions) to describe the data without systematically over- or underestimating any section of the curve, the number of runs of sign of the residuals was calculated (Motulsky and Ransnas, 1987). A run is a sequence of residuals with the same sign (positive or negative). For this test, the average residual of replicate observations was used for each incubation time. Several statistics, including mean square prediction error (MSPE), root of MSPE (rMSPE) expressed as a percentage of the observed mean (Theil 1966), coefficient of determination $\left(\mathrm{R}^{2}\right)$, Bayesian information criteria (BIC), and lack-of-fit test (Draper and Smith, 1998) were used to evaluate general goodness-of-fit (quality of prediction) of each model. Mean square prediction error was calculated as the sum of squared differences between observed and predicted values divided by the number of experimental observations. Root of MSPE was also calculated (MSPE divided by the observations mean) so that the MSPE could be expressed in the same units as the observed and predicted variables. The MSPE was decomposed into mean bias or error in central tendency (ECT), slope bias or error due to regression (ER), and random or error due to disturbance (ED). These 3 components were calculated as follows (Bibby and Toutenburg, 1977):

$$
\begin{aligned}
\mathrm{ECT} & =(\bar{P}-\bar{A})^{2} \\
\mathrm{ER} & =\left(S_{P}-r \times S_{A}\right)^{2} \\
\mathrm{ED} & =\left(1-r^{2}\right) \times S_{A}^{2}
\end{aligned}
$$

and expressed as a percentage of MSPE. The entities $\bar{P}$ and $\bar{A}$ are the averaged predicted and observed values, respectively; $S_{P}$ and $S_{A}$ are the standard deviations of the predicted and observed values, respectively; and $r$ is the coefficient of correlation between predicted and observed values. Error in central tendency indicates how the average of predicted values deviates from the average of observed values. Error due to regression (regression bias) measures deviation of the least squares regression coefficient $\left(r \times S_{A} / S_{P}\right)$ from 1 , the value it would have been if the predictions were completely accurate. When large, it indicates inadequacies in the ability of the model to predict the variable in question. Error due to disturbance represents the variation in observed values unexplained after the mean and the regression biases have been removed. The BIC was calculated using the nonlinear mixed procedure (PROC NLMIXED) of SAS (SAS Institute, 1999) and was used as another statistic for testing of the quality of fit. For the lack-of-fit test, percentage of curves with significant lack-of-fit within each version of each model was calculated. 

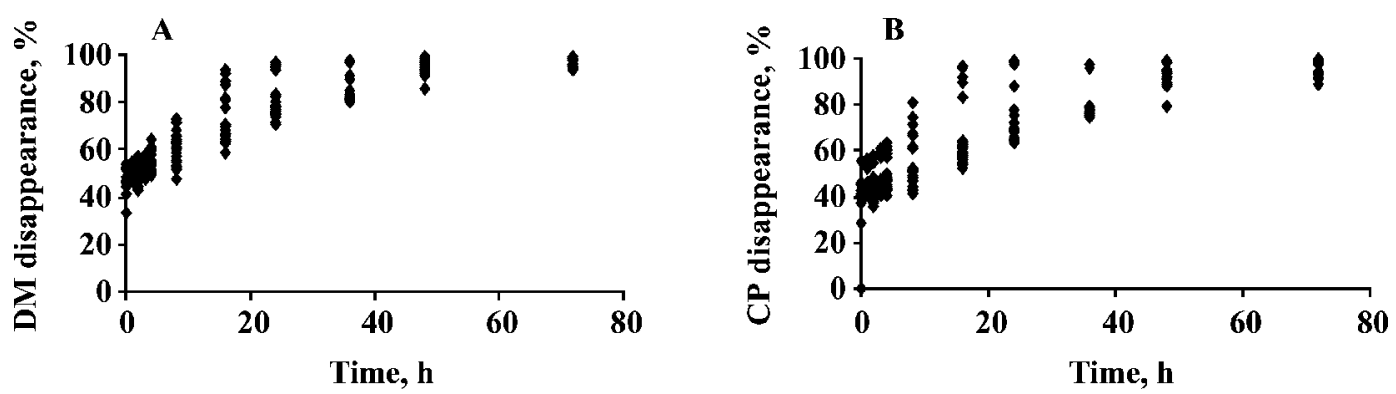

Figure 1. Plots of DM (A) and CP (B) in situ disappearance curves for all the experimental feeds.

\section{RESULTS AND DISCUSSION}

\section{Test Feeds}

Figure 1 shows plots of all the observed DM and CP disappearance data, including replicates, across the 6 feeds against incubation times. Variability in disappearance was related to washout fraction of the feeds and effect of heat processing on changing the fractional degradation rate and hence ruminal degradability pattern of the feeds, so that heat-processed soybeans had lower DM and CP disappearance (\%) over a given incubation time than raw soybeans. The effect of heat processing on the disappearance rate of highly degradable feeds is well known (NRC, 2001) and use of raw and roasted soybeans in this study allowed different models to be evaluated over a wider range of data.

\section{Model Behavior}

All curves could be fitted by the 3 models using nonlinear regression and utilizing the PROC NLIN of SAS (SAS Institute, 1999) because convergence to a solution occurred in all cases. Tables 3 to 5 show the estimated parameter values for models I, II, and III, respectively. With model III when unconstrained, $(a+b)$ tended to a value larger than 1 (for both $\mathrm{DM}$ and $\mathrm{CP}$ in the nonlagged version and $\mathrm{CP}$ in the lagged version), which is unacceptable biologically; therefore, the sum of these 2 parameters was constrained to be no greater than unity. The nonlagged versions reached the constraint limit, so the value of $U$ was zero with no associated error. For CP in the lagged version of model III, the solution was not met when restricted as above, so the constraint was lifted and solution was found with $(a+$ $b$ ) being slightly above 1 (but not significantly higher). In all nonlagged versions of the models, $L$ was constrained to be zero. In addition to the fitting problems observed when using model III, a larger number of iterations was required and greater sensitivity to starting values was observed, especially when the lagged form was used, which is symptomatic of an ill-conditioned or inappropriate model (Lopez et al., 1999). In spite of the fact that model I is a segmented model with 2 or 3 straight lines and needs a sufficient number of observations in each segment to obtain a consistent solution, and that model II is a exponential model, which is sometimes inadequate for describing ruminal disappearance curves (Dhanoa et al., 1995; Van Milgen and Baumont, 1995), the behavior of these 2 models demonstrated their suitability for fitting the DM and CP degradability data generated by our samples. Lopez et al. (1999) pointed out that disappearance of some feed components, particularly structural carbohydrates, exhibits a larger variety of forms than does CP. With some feeds, especially forages, the assumption that ruminal degradation follows zero-order or simple first-order kinetics may not be appropriate and therefore, sigmoidal models are more suitable.

\section{Statistical Evaluation}

There are different statistical tests for ranking and evaluating models. Sometimes results from these different tests seem contradictory, so an overall assessment is needed in this situation. The number of runs of sign of residuals, MSPE, rMSPE, $\mathrm{R}^{2}$, BIC, plot of residuals against predicted values and lack-of-fit test are the most widely used statistical criteria for comparing models. The number of runs of sign from fitting the lagged and nonlagged versions of each model did not tend to be different and this criterion could not resolve whether the lag parameter was necessary in each model (Table 6). However, between models, model III gave a high percentage of curves with 3 or fewer runs (for both DM and CP components) indicating that the residuals were not randomly distributed over the incubation times and this model was not as good as the other 2 for fitting data. In addition to parameters estimated, MSPE and $\mathrm{R}^{2}$ for each model are shown in Tables 3 to 5 . Each model was run with or without the lag for each disappearance curve for both DM and CP components. The $F$-values indicated that the more complex version (lagged) did 
Table 3. Parameter estimates for lagged and nonlagged versions of model I (SE in parentheses), and statistics calculated for comparison of the 2 equations

\begin{tabular}{|c|c|c|c|c|}
\hline \multirow[b]{2}{*}{ Item } & \multicolumn{2}{|c|}{ Lagged version } & \multicolumn{2}{|c|}{ Nonlagged version } \\
\hline & $\mathrm{DM}$ & $\mathrm{CP}$ & $\mathrm{DM}$ & $\mathrm{CP}$ \\
\hline \multicolumn{5}{|c|}{ Parameter estimates $^{1}$} \\
\hline$a$ & $50.27(2.523)$ & $43.64(4.686)$ & $47.96(1.464)$ & 43.07 (3.737) \\
\hline$b$ & $45.95(2.753)$ & $50.72(1.828)$ & $47.72(2.645)$ & $51.35(2.525)$ \\
\hline$c$ & $0.012(0.0027)$ & $0.013(0.0030)$ & $0.015(0.0078)$ & $0.016(0.092)$ \\
\hline$U$ & $3.79(2.241)$ & $5.64(3.606)$ & $4.32(1.423)$ & $5.58(3.084)$ \\
\hline$L$ & 1.30 (2.195) & $0.0\left(8.2 \times 10^{-7}\right)$ & - & - \\
\hline$E(k=0.06)$ & $73.40(4.206)$ & $69.31(6.584)$ & $74.07(4.820)$ & $70.63(8.658)$ \\
\hline$E(k=0.08)$ & $70.50(4.223)$ & $66.22(6.624)$ & $71.18(4.734)$ & $67.62(8.719)$ \\
\hline R-squared (\%) & $98.79(2.250)$ & $97.06(3.82)$ & $97.40(0.782)$ & $96.32(2.613)$ \\
\hline $\mathrm{rMSPE}^{2}$ & $4.17(1.341)$ & $5.36(1.795)$ & $4.36(0.666)$ & $5.66(1.894)$ \\
\hline \multicolumn{5}{|c|}{ MSPE analysis ${ }^{3}$ (\%MSPE) } \\
\hline ECT & $0.16(0.209)$ & $5.74(6.411)$ & $0.01(0.012)$ & $4.31(7.647)$ \\
\hline ER & $1.49(2.202)$ & $1.11(1.755)$ & $0.03(0.009)$ & $4.69(8.048)$ \\
\hline ED & $98.35(2.263)$ & $93.15(7.140)$ & $99.96(0.020)$ & $91.00(15.687)$ \\
\hline
\end{tabular}

${ }^{1} a=$ rapidly soluble fraction $(\%) ; b=$ slowly degradable fraction $(\%) ; c=$ degradation rate constant $(\% / \mathrm{h})$ $U=$ undegradable fraction (\%), calculated as $(1-a-b) ; L=$ lag time (h); $E=$ extent of degradation (\%); $k=$ fractional passage rate $(/ \mathrm{h})$.

${ }^{2}$ Root of mean square prediction error (\% observed mean).

${ }^{3} \mathrm{ECT}=$ Error in central tendency; ER = error due to regression; and ED = error due to disturbance.

not fit the data (disappearance curves for both DM and CP components) significantly better than the simpler (nonlagged) version (data not shown). In addition, the value of rMSPE was not significantly different between the 2 versions for both components and was sufficiently small to show that both forms are able to estimate model parameters accurately. Moreover, decomposition of MSPE gave similar values of ECT, ER, and ED for both versions of each model, and was mainly dominated by the disturbance component, which indicates that ruminal degradation of $\mathrm{DM}$ and $\mathrm{CP}$ of samples was well represented by both lagged and nonlagged forms. However, Mitchell and Sheehy (1997) argue that MSPE has limitations as an indicator of model performance because it weights the deviations by their squares, giving more influence to larger deviations. Therefore, the difference between parameter $L$ and zero was tested by $t$-test within each model $(P<0.05)$. No significant difference was found for any of 3 models, suggesting that the parameter was unnecessary in this case, so the

Table 4. Parameter estimates for lagged and nonlagged versions of model II (SE in parentheses), and statistics calculated for comparison of the 2 equations

\begin{tabular}{lccccc}
\hline & \multicolumn{2}{c}{ Lagged version } & & \multicolumn{2}{c}{ Nonlagged version } \\
\cline { 2 - 3 } \cline { 5 - 6 } Item & $\mathrm{DM}$ & $\mathrm{CP}$ & $\mathrm{DM}$ & $\mathrm{CP}$ \\
\hline Parameter estimates $^{1}$ & $49.04(3.331)$ & $45.03(4.454)$ & & $45.31(2.351)$ & $40.44(3.250)$ \\
$a$ & $49.34(4.611)$ & $51.73(3.449)$ & & $54.53(2.190)$ & $59.23(3.352)$ \\
$b$ & $0.057(0.0364)$ & $0.065(0.0386)$ & & $0.050(0.0269)$ & $0.051(0.0339)$ \\
$c$ & $1.63(1.481)$ & $3.24(2.362)$ & & $0.17(0.371)$ & $0.33(0.370)$ \\
$U$ & $2.43(4.256)$ & $2.17(4.115)$ & & & \\
$L$ & $70.97(7.250)$ & $64.57(10.880)$ & & $68.94(5.680)$ & $65.0(9.970)$ \\
$E(k=0.06)$ & $67.19(7.010)$ & $60.75(10.786)$ & & $65.40(5.483)$ & $61.36(9.874)$ \\
$E(k=0.08)$ & $97.47(0.910)$ & $96.38(1.671)$ & & $96.81(0.911)$ & $95.97(1.041)$ \\
R-squared $(\%)$ & $4.30(0.787)$ & $5.76(1.405)$ & & $4.85(0.543)$ & $6.17(0.865)$ \\
rMSPE ${ }^{2}$ & & & & \\
MSPE analysis ${ }^{3}$ (\%MSPE) & $0.11(0.271)$ & $0.72(1.636)$ & & $0.05(0.027)$ & $0.85(1.964)$ \\
ECT & $0.41(1.012)$ & $0.30(0.727)$ & & $0.21(0.322)$ & $1.49(2.929)$ \\
ER & $99.37(1.234)$ & $98.98(2.360)$ & & $99.74(0.380)$ & $97.60(4.866)$ \\
ED & &
\end{tabular}

${ }^{1} a=$ rapidly soluble fraction $(\%) ; b=$ slowly degradable fraction $(\%) ; c=$ fractional degradation rate $(/ \mathrm{h}) ;$ $U=$ undegradable fraction (\%), calculated as $(1-a-b) ; L=$ lag time (h); $E=$ extent of degradation (\%); $k=$ fractional passage rate $(/ \mathrm{h})$.

${ }^{2}$ Root of mean square prediction error (\% observed mean).

${ }^{3} \mathrm{ECT}=$ error in central tendency; $\mathrm{ER}=$ error due to regression; and $\mathrm{ED}=$ error due to disturbance. 
Table 5. Parameter estimates for lagged and nonlagged versions of model III (SE in parentheses), and statistics calculated for comparison of the 2 equations

\begin{tabular}{|c|c|c|c|c|}
\hline \multirow[b]{2}{*}{ Item } & \multicolumn{2}{|c|}{ Lagged version } & \multicolumn{2}{|c|}{ Nonlagged version } \\
\hline & $\mathrm{DM}$ & $\mathrm{CP}$ & $\mathrm{DM}$ & $\mathrm{CP}$ \\
\hline \multicolumn{5}{|c|}{ Parameter estimates $^{1}$} \\
\hline$a$ & $47.67(1.672)$ & $45.17(4.088)$ & $41.08(4.062)$ & $36.88(2.769)$ \\
\hline$b$ & $52.16(1.625)$ & $55.25(3.014)$ & $58.92(4.062)$ & $63.12(2.769)$ \\
\hline$U$ & $0.17(0.410)$ & $-0.42(1.578)$ & 0.00 & 0.00 \\
\hline$T$ & $11.80(3.784)$ & $13.83(5.562)$ & $12.99(5.732)$ & $15.60(7.216)$ \\
\hline $\bar{L}$ & $2.65(2.850)$ & $2.81(2.833)$ & - & - \\
\hline$E(k=0.06)$ & $64.23(8.786)$ & $58.46(9.937)$ & $69.29(4.839)$ & $65.17(7.856)$ \\
\hline$E(k=0.08)$ & $60.37(8.326)$ & $55.09(9.556)$ & $66.13(4.725)$ & $61.82(7.529)$ \\
\hline $\mathrm{R}$-squared (\%) & $93.18(4.041)$ & $93.20(5.491)$ & $92.64(1.054)$ & $91.94(1.046)$ \\
\hline $\mathrm{rMSPE}^{2}$ & $6.92(1.993)$ & $7.70(2.799)$ & $7.41(0.034)$ & $8.76(0.630)$ \\
\hline \multicolumn{5}{|c|}{ MSPE analysis ${ }^{3}(\% \mathrm{MSPE})$} \\
\hline ECT & $8.67(7.797)$ & $4.56(5.010)$ & $5.46(1.571)$ & $7.00(6.096)$ \\
\hline ER & $8.08(16.253)$ & $9.65(16.622)$ & $8.97(2.426)$ & $8.17(5.180)$ \\
\hline ED & $83.24(23.408)$ & $85.79(20.884)$ & $85.57(2.115)$ & $86.16(4.755)$ \\
\hline
\end{tabular}

${ }^{1} a=$ rapidly soluble fraction $(\%) ; b=$ slowly degradable fraction $(\%) ; U=$ undegradable fraction $(\%)$, calculated as $(1-a-b) ; T=$ time constant (h), such $L+T$ is the time taken half of the slowly degradable fraction to disappear; $L=$ lag time (h); $E=$ extent of degradation $(\%) ; k=$ fractional passage rate $(/ \mathrm{h})$.

${ }^{2}$ Root of mean square prediction error (\% observed mean).

${ }^{3} \mathrm{ECT}=$ error in central tendency; $\mathrm{ER}=$ error due to regression; and $\mathrm{ED}=$ error due to disturbance.

nonlagged version was chosen for comparing models. In contrast to Lopez et al. (1999), who concluded that inclusion of lag was a considerable improvement to the fit of nonsigmoidal models, our data were best described by nonlagged, nonsigmoidal models. This is because of a delay in disappearance at the beginning of incubation with forages, so a sigmoidal trend is more noticeable, and a lag is more likely. However, with a concentrate such as soybeans, disappearance is fast from the start of incubation and an extended lag is not observed.

The results of comparison between models are shown in Tables 7 and 8. After fitting each model to each disappearance curve, statistics were calculated. To conduct a multiple comparison, ANOVA was performed using PROC GLM of SAS (SAS Institute, 1999) to detect significant differences between models in MSPE, rMSPE, $\mathrm{R}^{2}$, and BIC values. The averages of statistics were then compared using the Duncan Multiple Range test. Root of MSPE as an indicator of model accuracy showed that models I and II gave significantly $(P<0.01)$ better fits to both feed components than model III. In addition, based on BIC values, models I and II showed the best fit to the data on DM disappearance, but models II and III were not significantly different for the CP data. The value of $R^{2}$ indicated that the proportion of variation explained was high for models I and II and that these models fit the DM and CP disappearance curves better than model III. The plot of residuals against predicted values is also useful visually and provides an easy to use approach for assessing model suitability that is valid for both linear and nonlinear regression (Draper and Smith, 1998). As Figure 2 shows, the horizontal band in plots for models I and II indicated no abnormality in residuals, but plots for model III indicated abnormality for both DM and CP, suggesting model inadequacy and the need for extra terms in the

Table 6. Percentage of curves (both DM and CP) for each number of runs of sign of the residuals observed when fitting lagged and nonlagged versions of each model ${ }^{1}$

\begin{tabular}{|c|c|c|c|c|c|c|c|c|c|c|c|c|}
\hline \multirow{3}{*}{$\begin{array}{l}\text { Number of } \\
\text { runs of sign }\end{array}$} & \multicolumn{4}{|c|}{ Model I } & \multicolumn{4}{|c|}{ Model II } & \multicolumn{4}{|c|}{ Model III } \\
\hline & \multicolumn{2}{|c|}{ Lagged } & \multicolumn{2}{|c|}{ Nonlagged } & \multicolumn{2}{|c|}{ Lagged } & \multicolumn{2}{|c|}{ Nonlagged } & \multicolumn{2}{|c|}{ Lagged } & \multicolumn{2}{|c|}{ Nonlagged } \\
\hline & $\mathrm{DM}$ & $\mathrm{CP}$ & $\mathrm{DM}$ & $\mathrm{CP}$ & $\mathrm{DM}$ & $\mathrm{CP}$ & $\mathrm{DM}$ & $\mathrm{CP}$ & $\mathrm{DM}$ & $\mathrm{CP}$ & $\mathrm{DM}$ & $\mathrm{CP}$ \\
\hline$\leq 3$ & 0.0 & 0.0 & 0.0 & 0.0 & 0.0 & 0.0 & 0.0 & 0.0 & 66.7 & 66.7 & 66.7 & 66.7 \\
\hline 4 & 0.0 & 0.0 & 0.0 & 16.7 & 0.0 & 0.0 & 0.0 & 16.7 & 16.7 & 33.3 & 33.3 & 0.0 \\
\hline 5 & 16.7 & 16.7 & 33.3 & 16.7 & 16.7 & 33.3 & 16.7 & 33.2 & 16.6 & 0.0 & 0.0 & 16.7 \\
\hline 6 & 66.7 & 16.7 & 33.3 & 16.7 & 16.7 & 16.7 & 33.3 & 16.7 & 0.0 & 0.0 & 0.0 & 16.6 \\
\hline$\geq 7$ & 16.6 & 66.6 & 33.4 & 50.0 & 66.6 & 50.0 & 50.0 & 33.4 & 0.0 & 0.0 & 0.0 & 0.0 \\
\hline
\end{tabular}

${ }^{1}$ Model I, zero-order degradation (France et al., 1990); model II, first-order degradation, constant $\mu$ (Ørskov and McDonald, 1979); model III, first-order degradation, variable $\mu$ (Lopez et al., 1999). 
Table 7. Comparison between models of goodness-of-fit and parameter estimates for DM degradability

\begin{tabular}{|c|c|c|c|c|c|}
\hline & Model I & Model II & Model III & SEM & $P$-value \\
\hline \multicolumn{6}{|c|}{ Statistical criteria ${ }^{1}$} \\
\hline R-squared (\%) & $97.40^{\mathrm{a}}$ & $96.81^{\mathrm{a}}$ & $92.64^{\mathrm{b}}$ & 0.038 & $* * *$ \\
\hline MSPE & $0.001^{\mathrm{b}}$ & $0.001^{b}$ & $0.003^{\mathrm{a}}$ & 0.000005 & $* * *$ \\
\hline rMSPE & $4.36^{\mathrm{b}}$ & $4.85^{\mathrm{b}}$ & $7.41^{\mathrm{a}}$ & 0.22 & $* * *$ \\
\hline $\mathrm{BIC}$ & $-46.90^{\mathrm{b}}$ & $-58.27^{\mathrm{b}}$ & $2.40^{\mathrm{a}}$ & 14.58 & $*$ \\
\hline \multicolumn{6}{|c|}{ Parameter estimates $^{2}$} \\
\hline$a$ & $47.96^{\mathrm{a}}$ & $45.31^{\mathrm{a}}$ & $41.08^{\mathrm{b}}$ & 1.16 & $* * *$ \\
\hline$b$ & $47.72^{\mathrm{c}}$ & $54.53^{\mathrm{b}}$ & $58.92^{\mathrm{a}}$ & 1.25 & $* * *$ \\
\hline$U$ & $4.32^{\mathrm{a}}$ & $0.17^{\mathrm{b}}$ & $0.00^{\mathrm{b}}$ & 0.35 & $* * *$ \\
\hline$E(k=0.06)$ & 74.07 & 68.94 & 69.29 & 2.09 & 0.188 \\
\hline$E(k=0.08)$ & 71.18 & 65.40 & 66.13 & 2.02 & 0.120 \\
\hline
\end{tabular}

${ }^{\mathrm{a}-\mathrm{c}}$ Means in the same row with no common superscript letters differ.

${ }^{1} \mathrm{MSPE}=$ mean square prediction error; $\mathrm{rMSPE}=$ root of MSPE; BIC $=$ Bayesian information criteria (smaller value means a better model).

${ }^{2} a=$ rapidly soluble fraction (\%); $b=$ slowly degradable fraction (\%); $U$ = undegradable fraction (\%), calculated as $(1-a-b) ; E=$ extent of degradation $(\%) ; k=$ fractional passage rate $(/ \mathrm{h})$.

$* P<0.05 ; * * * P<0.001$.

fitted equation (e.g., square or cross-product terms) or for transformation of the observations before analysis (Draper and Smith, 1998). Linear and quadratic relationships between residuals and predicted values were assessed and results (not reported) showed statistically significant linear and quadratic trends $(P<0.001)$ for model III, but not for models I and II. The lack-of-fit test results (Figure 3) showed that both versions of model I fit the DM and CP disappearance data well with less than $20 \%$ of curves showing significant lackof-fit. Model II showed about 35\% of the curves with significant lack-of-fit, but in the nonlagged CP analysis this lack-of-fit reached 50\%. Model III showed the largest percentage of curves with a significant lack-offit, which together with residual plots (Figure 2) confirmed inadequacy of this model for this particular study.
Van Milgen and Baumont (1995) pointed out that a greater number of incubation points per curve than recorded under normal experimental conditions might be necessary to compare different disappearance models. Lopez et al. (1999) found most statistical tests for goodness-of-fit are of limited value in comparing nonlinear models; however, our results showed that the selected statistical criteria could discriminate between models and based on the data set used in this study, models I and II were more suitable for fitting DM and CP disappearance curves than model III.

\section{Estimates of Degradation Parameters}

Results of the ANOVA performed to check whether the use of different models resulted in similar or different estimates of the DM and CP degradation parame-

Table 8. Comparison between models of goodness-of-fit and parameter estimates for CP degradability

\begin{tabular}{|c|c|c|c|c|c|}
\hline & Model I & Model II & Model III & SEM & $P$-value \\
\hline \multicolumn{6}{|c|}{ Statistical criteria $^{1}$} \\
\hline $\mathrm{R}$-squared (\%) & $96.32^{\mathrm{a}}$ & $95.97^{\mathrm{a}}$ & $91.94^{\mathrm{b}}$ & 0.007 & $* * *$ \\
\hline MSPE & 0.002 & 0.006 & 0.004 & 0.0003 & 0.521 \\
\hline rMSPE & $5.66^{\mathrm{b}}$ & $6.17^{\mathrm{b}}$ & $8.76^{\mathrm{a}}$ & 0.52 & $* *$ \\
\hline $\mathrm{BIC}$ & $-24.85^{\mathrm{b}}$ & $-8.4^{\mathrm{ab}}$ & $14.48^{\mathrm{a}}$ & 10.87 & * \\
\hline \multicolumn{6}{|c|}{ Parameter estimates $^{2}$} \\
\hline a & $43.07^{\mathrm{a}}$ & $40.44^{\mathrm{a}}$ & $36.88^{\mathrm{b}}$ & 1.368 & * \\
\hline$b$ & $51.35^{\mathrm{c}}$ & $59.23^{\mathrm{b}}$ & $63.12^{\mathrm{a}}$ & 1.220 & $* * *$ \\
\hline$U$ & $5.58^{\mathrm{a}}$ & $0.33^{\mathrm{b}}$ & $0.00^{\mathrm{b}}$ & 0.730 & $* * *$ \\
\hline$E(k=0.06)$ & 70.63 & 65.00 & 65.17 & 3.667 & 0.483 \\
\hline$E(k=0.08)$ & 67.62 & 61.36 & 61.82 & 3.648 & 0.418 \\
\hline
\end{tabular}

${ }^{\mathrm{a}-c}$ Means in the same row with no common superscript letters differ.

${ }^{1} \mathrm{MSPE}=$ mean square prediction error; $\mathrm{rMSPE}=$ root of MSPE; BIC = Bayesian information criteria (smaller value means a better model).

${ }^{2} a=$ rapidly soluble fraction (\%); $b=$ slowly degradable fraction (\%); $U$ = undegradable fraction (\%), calculated as $(1-a-b) ; E=$ extent of degradation $(\%) ; k=$ fractional passage rate $(/ \mathrm{h})$.

$* P<0.05 ; * * P<0.01 ; * * * P<0.001$. 

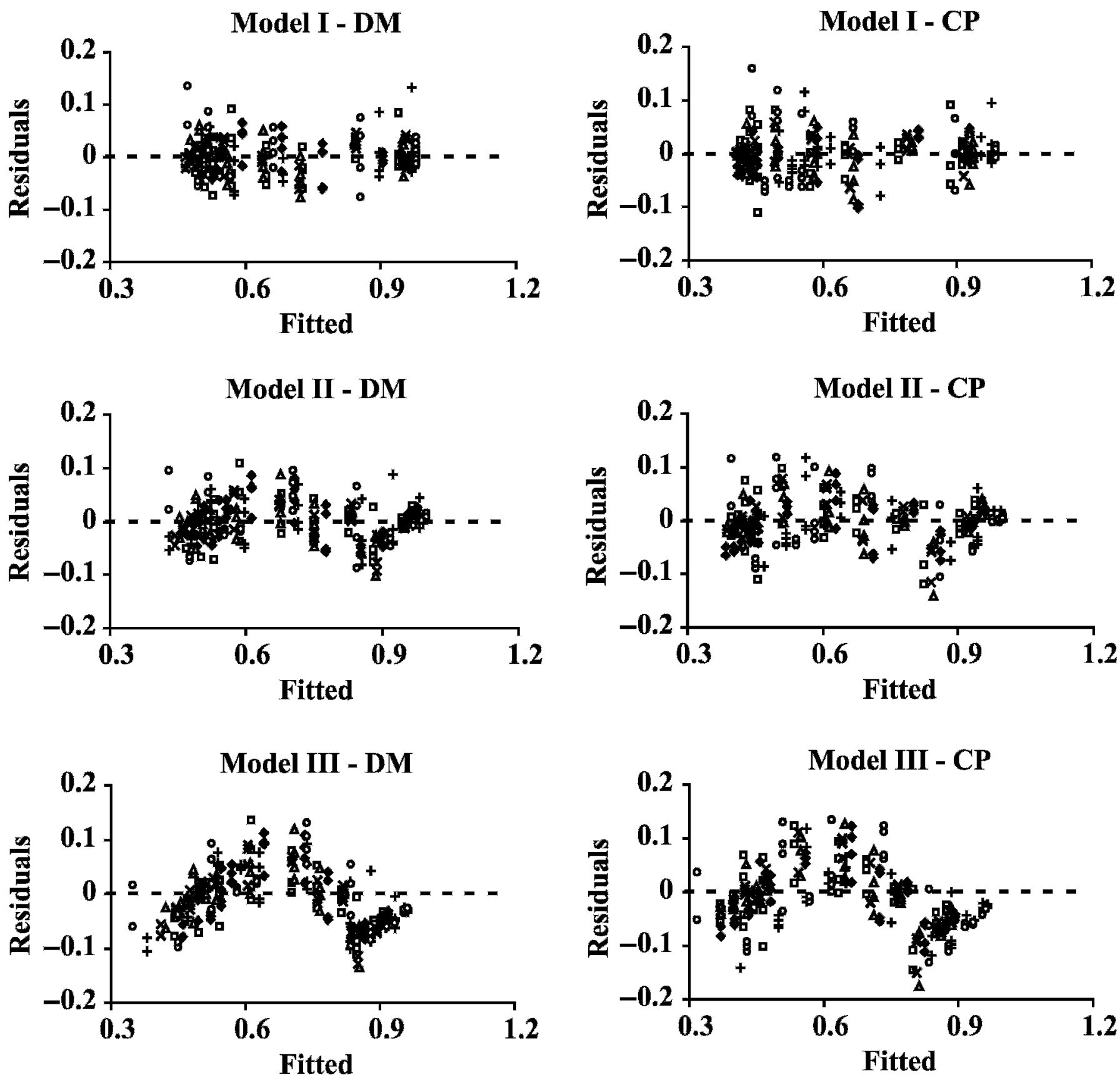

Figure 2. Plot of residuals against predicted values for DM and CP in situ disappearance curves for the 6 different feeds for model I, model II, and model III. Key: raw Sahar $(\diamond)$, roasted Sahar $(\triangle)$, steep-roasted Sahar $(\times)$, raw Williams $(+)$, roasted Williams $(\square)$, and steeproasted Williams $(\bullet)$ soybeans.

ters are shown in Tables 7 and 8. For DM and CP, estimates of the soluble fraction $(a)$ were lower with model III than with models I and II, but estimates of the insoluble potentially degradable fraction $(b)$ were higher with this model. Models II and III gave lower estimates of the DM and CP undegradable fractions $(U)$ than did model I. It is possible that these models underestimated that fraction, because the values obtained were much lower than the 72-h incubation residues. The $U$ value by definition needs to be no greater than the 72-h incubation residue value, because the $U$ is obtained at time infinity, so underestimation of the undegradable fraction with these models would be a drawback to their use. Estimates of the extent of degra- 


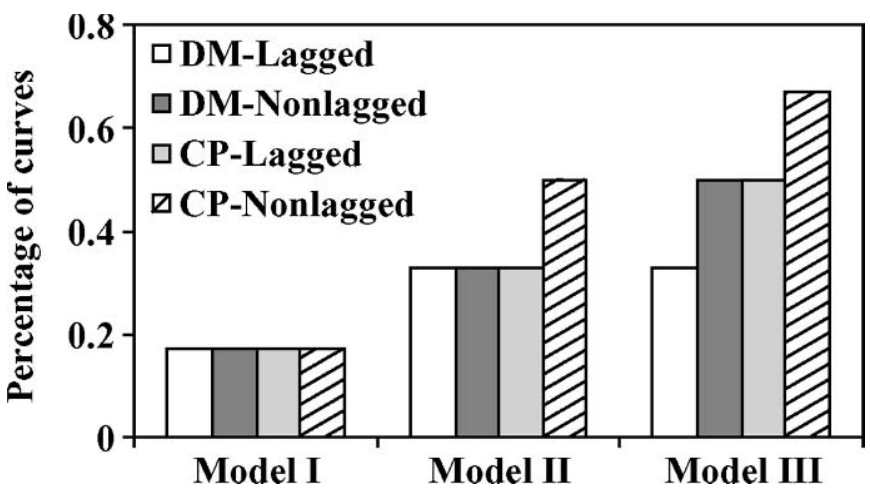

Figure 3. Percentage of curves with a significant lack-of-fit to DM and CP data for both lagged and nonlagged versions of models I, II, and III. Model I = zero-order degradation (France et al., 1990); model II = first-order degradation, constant $\mu$ (Ørskov and McDonald, 1979); model III = first-order degradation, variable $\mu$ (Lopez et al., 1999).

dation $(E)$ of DM and CP, which is the important entity, were higher at both passage rates of 0.06 and $0.08 / \mathrm{h}$ with model I than with models II and III. Although this numerical difference is of nutritional interest, it was not statistically significant. Results of the study by Lopez at al. (1999), in which different models were compared using incubation data for different forages, showed no significant differences between estimates of $E$ by the different models. However, differences in $E$ as determined by different models in the present study were larger than those observed by Lopez et al. (1999), probably due to different evaluated feeds, but in both studies the segmented model with up to 3 spline-lines showed higher estimates of $E$. It should be noted that the expression for $E$ for model III is an integral with no analytical solution, making the model slightly more difficult to use. Ability to obtain good estimates of all degradation parameters and ease of application are important in choice of a model.

\section{CONCLUSIONS}

To assess the validity of mathematical models to describe the degradation pattern of a given feed, their behavior, statistical performance, and biological characteristics should be evaluated. The results of this study based on various statistical tests showed that the piecewise linear and exponential models are well suited to describing the degradability patterns obtained for whole raw and roasted soybeans. In all the models tested, the parameter estimates for lag phase were not significant, suggesting that the nonlagged version of the models are suitable to describe degradation patterns in soybeans.

\section{REFERENCES}

Bibby, J., and H. Toutenburg. 1977. Prediction and Improved Estimation Models. John Wiley \& Sons, Chichester, UK.

Dhanoa, M. S., J. France, S. Lopez, J. Dijkstra, S. J. Lister, D. R. Davies, and A. Bannink. 1999. Correcting the calculation of extent of degradation to account for particulate matter loss at zero time when applying the polyester bag method. J. Anim. Sci. 77:3385-3391.

Dhanoa, M. S., J. France, R. C. Siddons, S. Lopez, and J. G. BuchananSmith. 1995. A non-linear compartmental model to describe forage degradation kinetics during incubation in polyester bags in the rumen. Br. J. Nutr. 73:3-15.

Draper, N. R., and H. Smith. 1998. Applied Regression Analysis, third edition. John Wiley \& Sons, New York, NY.

France, J., S. Lopez, E. Kebreab, A. Bannink, M. S. Dhanoa, and J. Dijkstra. 2005. A general compartmental model for interpreting gas production profiles. Anim. Feed Sci. Technol. 123-124:473485.

France, J., J. H. M. Thornley, S. Lopez, R. C. Siddons, M. S. Dhanoa, P. J. Van Soest, and M. Gill. 1990. On the two-compartment model for estimating extent of feed degradation in the rumen. J. Theor. Biol. 146:269-287.

Huntington, J. A., and D. I. Givens. 1995. The in situ technique for studying the rumen degradation of feeds: A review of the procedure. Nutr. Abstr. Rev. Ser. B 65:63-93.

Kebreab, E., J. A. N. Mills, L. A. Crompton, A. Bannink, J. Dijkstra, W. J. J. Gerrits, and J. France. 2004. An integrated mathematical model to evaluate nutrient partition in dairy cattle between the animal and its environment. Anim. Feed Sci. Technol. 112:131-154.

Lopez, L., J. France, M. S. Dhanoa, F. Mould, and J. Dijkstra. 1999. Comparison of mathematical models to describe disappearance curves obtained using the polyester bag technique for incubating feeds in the rumen. J. Anim. Sci. 77:1875-1888.

Mitchell, P. L., and J. E. Sheehy. 1997. Comparison of predictions and observations to assess model performance: A method of empirical validation. Pages 437-451 in Applications of Systems Approaches at the Field Level. M. J. Kropff, P. S. Teng, P. K. Aggarwal, J. Bouma, B. A. M. Bouman, J. W. Jones, and H. H Van Laar, ed. Kluwer Academic, Boston, MA.

Motulsky, H. J., and L. A. Ransnas. 1987. Fitting curves to data using nonlinear regression: A practical and nonmathematical review. FASEB J. 1:365-374.

National Research Council (NRC). 2001. Nutrient Requirements of Dairy Cattle. 7th rev. ed. National Academy of Science, Washington, DC.

Nocek, J. E. 1988. In situ and other methods to estimate ruminal protein and energy digestibility: A review. J. Dairy Sci. 71:2051-2069.

Ørskov, E. R., and I. McDonald. 1979. The estimation of protein degradability in the rumen from incubation measurements weighted according to rates of passage. J. Agric. Sci. (Camb.) 92:449-503.

SAS Institute. 1999. User's Guide: Statistics, 1999. Version 8.2. SAS Institute Inc., Cary, NC.

Theil, H. 1966. Applied Economic Forecasting. North-Holland Publishing Company, Amsterdam, The Netherlands.

Thomas, C. 2004. Feed into Milk - A new applied feeding system for dairy cows. Nottingham University Press, Nottingham, UK.

Thornley, J. H. M., and J. France. 2006. Mathematical Models in Agriculture. 2nd ed. CABI Publishing, Wallingford, UK.

Van Milgen, J., and R. Baumont. 1995. Models based on variable fractional digestion rates to describe ruminal in situ digestion. Br. J. Nutr. 73:793-807. 